\title{
Análisis bibliométrico de la producción científica peruana sobre la formación de profesionales de la salud
}

\author{
Dennis Arias Chávez ${ }^{1}$, Jessica Palacios-Garay ${ }^{2}$, Doris Fuster-Guillen ${ }^{3}$, Miguel Faustino-Sánchez ${ }^{3}$, César $^{3}$ \\ Borja-Villanueva ${ }^{4}$, Yolvi Ocaña-Fernández ${ }^{3}$, ${ }^{*}$ Ronald M. Hernández $^{5}$ \\ ${ }^{1}$ Universidad Continental, Arequipa Perú \\ ${ }^{2}$ Universidad Nacional Federico Villarreal, Lima, Perú \\ ${ }^{3}$ Universidad Nacional Mayor de San Marcos, Lima, Perú \\ ${ }^{4}$ Universidad Privada Juan Pablo II, Lima, Perú \\ ${ }^{5}$ Universidad San Ignacio de Loyola, Lima, Perú
}

Cómo referenciar este artículo/ How to reference this article:
Arias Chávez D, Palacios-Garay J, Fuster-Guillen D, Faustino-Sánchez $M$, Borja-Villanueva $C$, Ocaña-Fernández $\mathbf{Y}$, et al. Análisis bibliométrico de la producción científica peruana sobre la formación de profesionales de la salud. Mem. Inst. Investig. Cienc. Salud. 2019; 17(3): 41-48

\section{RE S U M E N}

La investigación y la publicación científica son aspectos importantes dentro de la formación profesional en salud, pues permitirán practicar eficientemente una medicina basada en evidencias. El objetivo del trabajo fue describir a través de indicadores bibliométricos la producción científica relacionada con la formación de profesionales de la salud en Perú durante el periodo 2014-2018. Estudio bibliométrico sobre la totalidad de la producción científica peruana que trata de la formación de profesionales de la salud en el período 20142018, recuperada de la base de datos de Google Académico. Se calcularon los indicadores cienciométricos de 155 artículos filtrados por el software Harzing's Publish or Perish v. 6. Las variables estudiadas fueron: total de artículos, citas, promedio de citas por año, por artículos, por autores y por autores por año, promedio de artículos por autor, promedio de autores por artículo, índices h, g, h contemporáneo (hc), h individual (hI), hI normalizado, AWCR, AW, AWCRpA, e, hm, hI anual, amplitud $\mathrm{H}$, amplitud, cantidad de autores, índice de colaboración, artículos más citados y distribución anual de los artículos, así como su publicador. Se obtuvo un total de 905 citas con un promedio de $181.00 ; 5.84$ y 457.37 citas por año, artículos y autores respectivamente. Las publicaciones poseen un índice $\mathrm{h}$ de 16 y un índice g de 19. Destaca la producción anual del año 2015, con 41 publicaciones. La mayor cantidad de autores trabajan en parejas (48 artículos). Se totalizaron 458 autores en los 155 artículos, generando un índice de colaboración de 2.95. La producción científica relacionada con la formación de profesionales de la salud en Perú fue escasa en los últimos 5 años. Los indicadores cienciométricos muestran una tendencia decreciente.

Palabras clave: Bibliometría, Producción Científica Peruana, Formación de Profesionales de la salud, Perú.

\section{Bibliometric analysis of Peruvian scientific production on the training of health professionals}

\section{A B S T R A C T}

Research and scientific publication are important aspects of professional training in health, as they will allow efficient practice of evidence-based medicine. The objective was to describe through bibliometric indicators the scientific production related to the training of health professionals in Peru during the period 2014-2018. Bibliometric study of the entire Peruvian scientific production that deals with the training of health professionals in the period 2014-2018, retrieved from the Google academic database. We calculated the scientometric indicators of 155 articles filtered by the Harzing's Publish or Perish v. 6 
software. The variables studied were: total number of articles, citations, average citations per year for articles, authors and authors per year, average articles per author, average number of authors per article, the $h, g$ indexes contemporary $h$ (hc), individual $h(h I)$, standard hI, AWCR, AW, AWCRpA, e, hm, annual hI, amplitude $\mathrm{H}$, amplitude, number of authors, index of collaboration, most-cited articles and annual distribution of articles, as well as their publishers. A total of 905 citations were obtained with an average of 181.00; 5.84 and 457.37 citations per year, articles and authors respectively. The publications have an $\mathrm{H}$-index of 16 and a G-index of 19 . The annual production of 2015, with 41 publications, stood out, the largest number of authors work in pairs (48 articles). A total of 458 authors were published in the 155 articles, generating a 2.95 collaboration rate. Scientific production related to the training of health professionals in Peru was scarce in the last 5 years. Scientometric indicators show a decreasing trend.

Keywords: Bibliometry, Peruvian Scientific Production, Training of Health Professionals, Peru.

\section{INTRODUCCIÓN}

El conocimiento científico es el motor de las grandes transformaciones que suceden en el mundo. El progreso de una nación se ve regida por alcanzar tres factores fundamentales: ciencia, tecnología e innovación. Su cumplimiento hace que las sociedades sean más prósperas y sostenibles ya que su impacto no solo se ve en el aspecto político o económico, sino también en lo social, medioambiental $y$, desde luego, en la calidad de vida de las personas $^{(1)}$. En este sentido, la labor investigativa apunta no solo a cumplir cuestiones metodológicas válidas, sino a garantizar que el conocimiento generado de ella sea sólidamente establecido(2). Esto se logra publicando los hallazgos ${ }^{(3,4)}$ en revistas especializadas con el fin de cerrar el círculo comunicativo que exige la labor científica.

El análisis bibliométrico permite observar tendencias dentro de la literatura científica, extraer conclusiones sobre el impacto del material publicado y, lo que es más importante, dirigir los esfuerzos de investigación y financiación de una manera más informada. Esta disciplina se vale del meta-análisis de grandes cantidades de bibliografía con el fin de identificar, no solo patrones, sino también determinar relaciones, tendencias e indicadores a partir de la información científica producida y almacenada en bases de datos. Sus esfuerzos se orientan a proporcionar información relevante y válida sobre los actores y procesos desarrollados en la investigación científica, tomando como objeto de estudio los artículos científicos publicados en las revistas especializadas (científicas). Al respecto, es importante destacar el papel que cumplen estos productos como insumos primarios del quehacer científico. La dinámica de la publicación ha cambiado gracias a la aparición de los medios electrónicos que han permitido que el proceso de edición, publicación y divulgación sea más eficiente, personalizada, masiva, actualizada y abierta, lo que, en cuestiones de recursos significa también un mayor ahorro de costos y tiempo para el análisis de la calidad de los mismos $^{(5)}$.

La investigación en salud es considerada un proceso dirigido a generar conocimiento sistemático y contrastar hipótesis para mejorar la salud y bienestar de la población ${ }^{(6)}$. Para la Organización Panamericana de la Salud (OPS) los sistemas nacionales de investigación en salud son aquellos constituidos por los individuos e instituciones que rigen, administran, coordinan, requieren, crean, comunican o usan los resultados de una investigación para promover, restablecer, mejorar o mantener el estado de salud ${ }^{(7)}$. El resultado de toda investigación debería terminar en una publicación, que permita tomar decisiones y usar el conocimiento para la mejora del desarrollo de una población. Sin embargo, en nuestro país no es común que esto suceda esto, la problemática va relacionada con la poca capacitación en redacción de artículos y una ausencia de cultura de publicación ${ }^{(8)}$; situación que se ha venido revirtiendo a través de diferentes cursos realizados a nivel nacional. A nivel latinoamericano, podemos mencionar que los factores que intervienen en el proceso de formación de los profesionales de la salud, son complejos y cada uno de ellos requiere el máximo de atención institucional, de los centros docentes y unidades de la salud, de sus dirigentes y profesores, así como la dedicación permanente de los estudiantes al estudio, con el fin de lograr egresados de alto nivel político-ideológico, técnico, científico y humanista ${ }^{(9)}$.

En los últimos años, el interés por los estudios bibliométricos han aumentado. Estos se han ido orientando no solo a evaluar la literatura científica en revistas propiamente dichas, 
sino también en campos específicos de la actividad médica. Sin embargo, son escasos los estudios focalizados en producción científica peruana sobre la formación de profesionales de la salud, razón que motivó la realización del presente artículo, y cuyo objetivo es describir, a través de indicadores bibliométricos, la producción científica relacionada con la formación de profesionales de la salud en Perú durante el periodo 2014-2018.

\section{MATERIALES Y MÉTODOS \\ Diseño y unidades de análisis}

Se realizó un estudio bibliométrico descriptivo-retrospectivo sobre la totalidad de la producción científica peruana que trata sobre la formación de profesionales de la salud en el período 2014-2018. Para el análisis de la información se utilizó la bibliometría, que es una gama de medidas cuantitativas que evalúan el impacto de los resultados de la investigación. Respecto a los indicadores bibliométricos, se utilizan para precisar la productividad de los autores o instituciones, medida por el número de sus trabajos, el crecimiento de cualquier campo de la ciencia, la variación cronológica del número de trabajos publicados, la colaboración entre los científicos o instituciones y el número de autores por trabajo o centros de investigación que colaboran ${ }^{(10)}$.

\section{Variables de estudio}

Las variables estudiadas fueron: total de artículos, citas, promedio de citas por año, promedio de citas por artículos, promedio de citas por autores, promedio de citas por autores por año, promedio de artículos por autor, promedio de autores por artículo, índice $\mathrm{h}$, índice $\mathrm{g}$, índice $\mathrm{h}$ contemporáneo (hc), índice $\mathrm{h}$ individual (hI), índice hI normalizado, índice AWCR, índice AW, índice AWCRpA, índice $\mathrm{e}^{(11)}$, índice $\mathrm{hm}$, índice hI anual, amplitud $\mathrm{H}$, amplitud, cantidad de autores, índice de colaboración, artículos más citados y distribución anual de los artículos, así como su publicador.

\section{Procedimientos, recolección y manejo de datos}

Tomando como base de datos el Google Académico, se calcularon los indicadores cienciométricos a través del software Harzing's Publish or Perish versión 6 , el cual mostró 946 resultados de la búsqueda "formación de profesionales de salud Perú". Luego se filtraron los resultados revisándolos de manera individual y descartando los que no cumplen la condición de ser los resultados de una investigación peruana, resultando al final 155 artículos. Los datos obtenidos se exportaron a una base de datos (MS Excel de Microsoft Corp) y los resultados se presentan en tablas con el fin de abordar el objetivo planteado.

\section{RESULTADOS}

\section{Indicadores Cienciométricos}

En la Tabla 1, se analizaron 155 artículos referentes a la producción científica peruana sobre la formación de profesionales de la salud correspondientes al período 2014-2018. Se obtuvo un total de 905 citas, con un promedio de 181.00 ; 5.84 y 457.37 citas por año, artículos y autores respectivamente. Las publicaciones poseen un índice $\mathrm{h}$ de 16 y un índice g de 19. 
Tabla 1: Indicadores cienciométricos

\begin{tabular}{ll}
\hline Años de publicación: & $\mathbf{2 0 1 4 - 2 0 1 8}$ \\
\hline Artículos: & 155 \\
Citas: & 905 \\
Citas/año: & 181.00 \\
Citas/artículo: & 5.84 \\
Citas/autor: & 457.37 \\
Artículos/autor: & 86.15 \\
Autores/artículo: & 2.32 \\
Índice-h: & 16 \\
Índice-g: & 19 \\
Índice h normal: & 9 \\
Índice hc & 17 \\
Índice hI & 5.95 \\
hl.anual: & 1.80 \\
AWCR & 260.32 \\
Índice AW & 16.13 \\
AWCRpA & 129.99 \\
Índice-e & 8.89 \\
Índice hm & 12.32 \\
Amplitud $\mathrm{h}$ & 37 \\
Amplitud g & 42.1 \\
\hline
\end{tabular}

\section{Artículos citados}

La Tabla 2 muestra la relación de los artículos más citados publicados en el período analizado, destacándose el de Taype Rondán, J. Huaccho Rojas, R. Pereyra Elías, Christian R. Mejía y Percy Mayta-Tristán sobre las "Características de los cursos de investigación en escuelas de medicina del Perú", que ha recibido 27 citas desde su publicación en el 2015, con un promedio de 6.75 citas por año.

Tabla 2: Diez artículos más citados de la producción Científica peruana sobre la formación de profesionales de la salud

\begin{tabular}{|c|c|c|c|}
\hline Autores & Título & Citas & Citas por año \\
\hline Á. Taype Rondán, J. Huaccho & Características de los cursos & & \\
\hline $\begin{array}{l}\text { Rojas, R. Pereyra Elías, Christian R. } \\
\text { Mejía, Percy Mayta Tristán }\end{array}$ & $\begin{array}{l}\text { de investigación en escuelas } \\
\text { de medicina del Perú }\end{array}$ & 27 & 6.75 \\
\hline $\begin{array}{l}\text { IE Corrales-Reyes, MJR García, JR } \\
\text { Pérez, MG Raga }\end{array}$ & $\begin{array}{l}\text { Limitantes de la producción } \\
\text { científica estudiantil } \\
\text { Nueva normativa de titulación }\end{array}$ & 24 & 12 \\
\hline R Valle, A Perales & $\begin{array}{l}\text { en el residentado médico en el } \\
\text { Perú: problemas y } \\
\text { perspectivas }\end{array}$ & 24 & 8 \\
\hline J Domínguez Granda & $\begin{array}{l}\text { La educación a distancia en el } \\
\text { Perú }\end{array}$ & 24 & 8 \\
\hline & $\begin{array}{l}\text { Participación en sociedades } \\
\text { científicas estudiantiles y en } \\
\text { cursos extracurriculares de }\end{array}$ & & \\
\hline $\begin{array}{l}\text { Carlos J. Toro-Huamanchumo, } \\
\text { Virgilio E. Failoc-Rojas y Cristian } \\
\text { Díaz-Vélez }\end{array}$ & $\begin{array}{l}\text { cursos extracurriculares de } \\
\text { investigación, asociados a la } \\
\text { producción científica de }\end{array}$ & 23 & 5.75 \\
\hline & $\begin{array}{l}\text { estudiantes de medicina } \\
\text { humana: estudio preliminar } \\
\text { Síndrome de burnout en }\end{array}$ & & \\
\hline M Picasso-Pozo & $\begin{array}{l}\text { estudiantes de Odontología de } \\
\text { una universidad peruana }\end{array}$ & 23 & 5.75 \\
\hline $\begin{array}{l}\text { Christian R. Mejía, Fiorella Inga- } \\
\text { Berrospi, Percy Mayta-Tristán }\end{array}$ & $\begin{array}{l}\text { Titulación por tesis en escuelas } \\
\text { de medicina de Lima, 2011: } \\
\text { características, motivaciones y } \\
\text { percepciones (Publicado 2014) }\end{array}$ & 23 & 4.6 \\
\hline
\end{tabular}


Fernando Munayco-Guillén Anais Cámara-Reyes L. Jaime MuñozTafur Hugo Arroyo-Hernández Christian R. Mejía Félix Lem-Arce Ubaldo E Miranda-Soberón

Elsy Miní, Julio Medina, Verónica Peralta, Luis Rojas, Joece Butron, Ericson L. Gutiérrez.

D Champin

Manuel Heredia Alarcón, Marcelino Andía Ticona, Helder Ocampo Guabloche, José Ramos-Castillo, Amado Rodríguez Caldas, Catalina Tenorio, Karim Pardo Ruiz
Características del maltrato hacia estudiantes de medicina de una universidad pública del 22 7.33 Perú

Programa de residentado médico: percepciones de los médicos residentes en

hospitales de Lima y Callao

Evaluación por competencias en la educación médica

\section{Deserción estudiantil en las} carreras de ciencias de la salud en el Perú

\section{Producción por años}

La Tabla 3 muestra la producción por años, destacando el año 2015, con 41 publicaciones, seguida del año 2014, con 38 publicaciones y del año 2017 con 34 publicaciones.

Tabla 3: Producción por años

\begin{tabular}{lll}
\hline Año & $\mathbf{f i}$ & \% \\
\hline 2014 & 38 & $24.5 \%$ \\
2015 & 41 & $26.5 \%$ \\
2016 & 36 & $23.2 \%$ \\
2017 & 34 & $21.9 \%$ \\
2018 & 6 & $3.9 \%$ \\
Total & 155 & $100.0 \%$ \\
\hline
\end{tabular}

\section{Bases de Datos}

La Tabla 4 muestra que la biblioteca electrónica SciELO, en sus diversas variantes, es la base de datos que aloja la mayor cantidad de publicaciones científicas referidas a la producción científica relacionada con la formación de profesionales de la salud en Perú con 81 resultados.

Tabla 4: Base de datos

\begin{tabular}{lll}
\hline Publicador & $\mathbf{f i}$ & $\mathbf{\%}$ \\
\hline Scielo & 81 & $52.26 \%$ \\
Otros & 19 & $12.26 \%$ \\
Repositorios de tesis & 19 & $12.26 \%$ \\
Revistas científicas & 17 & $10.97 \%$ \\
Elsevier & 7 & $4.52 \%$ \\
Redalyc & 5 & $3.23 \%$ \\
Dialnet & 5 & $3.23 \%$ \\
Researchgate & 2 & $1.29 \%$ \\
Total & 155 & $100.00 \%$ \\
\hline
\end{tabular}

\section{Distribución de autores firmantes}

La Tabla 5 muestra la distribución de autores firmantes, destacando que la mayor cantidad de autores trabajan en parejas (48 artículos) seguido de los autores individuales (43 artículos). Se totalizaron 458 autores en los 155 artículos, generando un índice de colaboración de 2.95 . 
Tabla 5: Distribución de autores firmantes

\begin{tabular}{lll}
\hline Autores & $\mathbf{f i}$ & $\mathbf{\%}$ \\
\hline 1 & 43 & $27.74 \%$ \\
2 & 48 & $30.97 \%$ \\
3 & 23 & $14.84 \%$ \\
4 & 15 & $9.68 \%$ \\
5 & 12 & $7.74 \%$ \\
Más de 5 & 14 & $9.03 \%$ \\
Total & 155 & $100.00 \%$ \\
\hline
\end{tabular}

\section{DISCUSIÓN}

La bibliometría como método cuantitativo permite examinar la estructura de conocimiento y el desarrollo de campos de investigación basados en el análisis de publicaciones relacionadas con éstas. Para lograr este objetivo se extraen y manipulan datos, utilizando métodos bibliométricos basados en el contenido o en el análisis de citas ${ }^{(12)}$. Estos métodos se han beneficiado enormemente del tratamiento informatizado de datos y en los últimos años ha habido un gran aumento en el número de publicaciones en este campo. Esto se debe en parte a los métodos computarizados, pero también al hecho de que un método bibliométrico debe incluir un cierto volumen de datos con el fin de que los resultados sean estadísticamente confiables.

La evaluación del comportamiento de la producción científica peruana sobre la formación de profesionales de la salud a través de un estudio bibliométrico de las publicaciones demuestra las características de esta actividad hasta la fecha y su tendencia en los próximos años; convirtiéndose además en una herramienta de consulta para los investigadores en este campo considerando que no abundan estudios similares al respecto.

Los indicadores cienciométricos de la presente investigación son inferiores, en cuanto al índice $\mathrm{h}^{(13)}$ y $\mathrm{g}$ a los encontrados en la investigación bibliométrica desarrollada para la producción científica de la revista Medisur ${ }^{(14)}$. Sin embargo, en el análisis de las citas la presente investigación es superior a la mencionada anteriormente. En este sentido, debe destacarse que el tema (la formación de profesionales de la salud) ha recibido 905 citas, con un promedio de 181.00 citas por año. Esto puede atribuirse a la importancia del tema y a que los artículos encontrados presentan una indexación en variadas bases de datos que cuentan con prestigio internacional y son fuentes de consulta de muchos investigadores.

En la presente investigación se observó que, en cuanto a la publicación anual, su tendencia es decreciente, destacando la del año 2015, con 41 publicaciones, seguida del año 2014, con 38 publicaciones y del año 2017 con 34 publicaciones. Este resultado discrepa de la investigación bibliométrica de los artículos sobre cuidado de enfermería publicada en revistas colombianas en donde se observa un incremento en la producción científica de Enfermería en Colombia, predominando la publicación de artículos originales ${ }^{(15)}$. Esto puede deberse al adecuado planteamiento de los objetivos propuestos por la Misión Ciencia Educación y Desarrollo, donde se buscaba preparar a Colombia para ponerse a la altura de los países en desarrollo, estableciendo políticas de transformación del conocimiento y de la educación para obtener científicos que impulsaran el desarrollo tecnológico y científico del país ${ }^{(16)}$.

Al analizar los artículos más citados, se observó que en el primer lugar se encuentra el estudio realizado por Á. Taype Rondán, J. Huaccho Rojas, R. Pereyra Elías, Christian R. Mejía, Percy Mayta-Tristán ${ }^{(17)}$ sobre las "Características de los cursos de investigación en escuelas de medicina del Perú". Constituye un aspecto llamativo que la mayoría de los diez artículos más citados son de auditoría peruana, destacando los autores Christian R. Mejía ${ }^{18-}$ 22) y Percy Mayta-Tristán ${ }^{(23,24)}$. Los críticos de los análisis bibliométricos afirman que los artículos no se citan porque representan la mejor calidad académica, sino para referirse a los resultados de un trabajo anterior o un método, para dar crédito a los resultados parciales hacia el mismo objetivo, para respaldar alguna terminología, para proporcionar antecedentes, leer ideas menos familiares $y$, a veces, criticar una determinada conclusión. Por ejemplo, el artículo más citado en la revisión bibliométrica con 27 citas hace referencia a las características de los cursos de investigación en escuelas de medicina del 
Perú. Este trabajo puede ser altamente citado en otras publicaciones, o se podría argumentar que este paper se cita regularmente ya que puede tomarse como un antecedente de calidad $y$, por lo tanto, puede tomarse como una fuente de consulta al realizar la introducción de otro artículo referido al tema ${ }^{(25)}$.

En lo que respecta a la distribución de autores con respecto al total de artículos publicados, el análisis indica que existe un predominio de papers escritos por dos autores, lo cual difiere con lo encontrado en la investigación desarrollada por Ramos, González-Alcaide y Gutiérrez, en la cual se observó predominio de artículos escritos por un único autor. La media de autores por documento fue de $2.95^{(26)}$. Puede entenderse que la colaboración científica es una considerada una vía apropiada para para obtener mayores y mejores resultados en la investigación en salud como para aumentar sus niveles de citación.

Es preciso indicar que entre las limitaciones del presente trabajo se encuentra la relacionada con las restricciones de los estudios bibliométricos y de los sesgos de la base de datos bibliográfica elegida para la recuperación de los documentos: Google Académico. No obstante, se eligió precisamente esta base de datos porque permite encontrar contenido netamente académico de un tema específico, en este caso, producción científica peruana sobre la formación de profesionales de la salud. Los resultados son generados de fuentes académicas comprobadas y relevantes. Además, muestra información nueva relacionada con los intereses buscados. Finalmente, incluye material de repositorios institucionales, a cuyas versiones web los usuarios pueden acceder de manera gratuita, generalmente a artículos que las bases de datos no tienden a enlazar.

Otra limitación del estudio se relaciona con la posibilidad de que existan otras investigaciones publicadas sobre el tema tratado entre los años 2014-2018, las cuales no fueron estudiadas por no encontrarse en la base de datos, o porque en el título, resumen y/o palabras claves no contenían las palabras usadas en el criterio de búsqueda. Lo mismo ocurre con el nombre de los autores, ya que el software en ocasiones no reconoce a la totalidad de autores, debiendo verificarse este aspecto de manera manual.

A partir de los hallazgos aquí recolectados se recomienda producir más investigaciones referidas al tema, al observarse que la actual no es abundante y deja aún muchos aspectos para la investigación, en pro de la mejora de la formación de profesionales de la salud en el Perú.

Para concluir, los profesionales del sector salud necesitan reforzar el desarrollo de las competencias investigativas, así como con la de motivación y el conocimiento por publicar, por tanto de aquí se deriva las inquietudes relacionadas con la formación profesional la cual debe buscar las competencias de promoción y reconocimiento a la investigación científica. El reto está planteado, avanzar en este campo dependerá no solo de las políticas y estrategias que se planteen desde las instituciones formativas, sino de la actitud para asumir un nuevo rol, aprender, investigar, publicar, enseñar y comunicar, como eje fundamental de su profesión.

\section{REFERENCIAS BIBLIOGRÁFICAS}

1. Soriano Álvarez C. Investigación científicomédica en el Perú: nuevos espacios. Rev Gatroenterol Peru. 2013; 33(3): 207-15.

2. Corrales-Reyes I, Fornaris-Cedeno $Y$, Reyes-Pérez J. Análisis bibliométrico de la revista investigación en educación médica. Período 2012-2016. Inv Ed Med. 2017; 7(25): 18-26.

3. Chaple AM. Fotografía clínica estomatológica: consejos para la práctica diaria. Rev Cub Estomatol. 2015; 52: 80-3.

4. Corrales IE, Reyes J]. Visibilidad científica de la revista "16 de Abril". Actualidad y perspectivas. 2015; 54(259): 1-3.

5. Michán L, Muñoz-Velasco I. Cienciometría para ciencias médicas: definiciones, aplicaciones y perspectivas. Inv Ed Med. 2013; 2(6): 100-6.

6. Fiestas F. La investigación como herramienta para mejorar el área de la salud mental y neurológica en países con

pocos recursos. Rev Neuropsiquiatr. 2009; 72(1-4): 47-57.

7. Gutierrez C, Mayta P. Publicación desde el pre grado en Latinoamérica: importancia, limitaciones y alternativas de solución. CIMEL. 2003; 8(1): 54-60.

8. Maz-Machado A, Jiménez-Fanjul N, Villarraga-Rico E. La producción científica colombiana en SciELO: un análisis bibliométrico. Revista Interamericana de Bibliotecología. 2016; 39(2), 111-9.

9. Carreño de Celis R, Salgado González L, Fernández Oliva B, Alonso Pardo ME. Factores que intervienen en el proceso de formación de los profesionales universitarios de la salud. Educ Médica Super. 2009; 23(3): 82-95.

10. Sancho R. Indicadores bibliométricos utilizados en la ciencia y tecnología. Rev Doc Cient. 1990; 13: 842-65. 
11. Chun-Ting Z. El índice electrónico, que complementa el índice h para citas en exceso. PLoS ONE. 2009; 5(5): e5429.

12. Wallin JA. Métodos bibliométricos: trampas y posibilidades. Farmacología básica y clínica y toxicología. 2005; 97(5): 261-75.

13. Egghe $L$, Rousseau R. Un modelo informétrico para el índice de Hirsch, Scientometrics. 2006; 69(1):121-30.

14. Corrales Reyes I, Fornaris Cedeño Y, Reyes Pérez J, Valdés Gamboa L. Aproximación cienciométrica a la producción científica de MediSur. MediSur. 2007; 15(5): 640-6.

15. Camacho D, Oviedo C, Ramos E, González T. Análisis bibliométrico de los artículos sobre cuidado de enfermería publicados en revistas colombianas. Rev Elect Enf Global. 2016; 44: 396-405.

16. Cañedo A, Nodarse R, Cruz F, Germán H, Celorrio I, Guerrero Pupo JC. Papel de la colaboración científica en el impacto de la investigación en salud de Cuba en el contexto de América Latina. Rev Cubana Sal Públ. 2016; 27(1): 56-74.

17. Taype-Rondán Á, Inga-Berrospi F, Casiano das $F$. Percepción de médicos recién egresados sobre las habilidades clínicas adquiridas durante el pregrado en Lima, Perú. Rev Med. 2015; 143(4): 540-42.

18. Mejia CR, Inga-Berrospi F, Mayta-Tristán P. Titulación por tesis en escuelas de medicina de Lima, 2011: características, motivaciones y percepciones. Rev Peru Med Exp Sal Pub. 2014; 31(3): 509-23.

19. Mejía C, Quiñones D, Chacón J, Aguirre A, Miñan TAL, Rosas VAM. Médicos investigadores: Percepción de estudiantes de medicina y factores asociados a la posibilidad de serlo. Educ Med Super. 2017; 31(3): 53-63.
20. Mejía C, Valladares-Garrido M, Luyo-Rivas A, Valladares-Garrido D, Talledo-Ulfe L, Vilela- Estrada MA. Factores asociados al uso regular de fuentes de información en estudiantes de medicina de cuatro ciudades del Perú. Rev Peru Med Exp Sal Pub. 2015; 32(2): 230-36.

21. Mejia C, Valladares-Garrido M, CvetkovicVega A, Inga-Berrospi F. Documentos de gestión en las sociedades científicas de estudiantes de medicina de Latinoamérica, 2014. Acta Med Peru. 2016; 33(1): 29-34.

22. Mejía C, Valladares-Garrido $M$, Alledo-Ulfe L, Sánchez-Arteaga K, Rojas C, RuizArimuya JJ. Síndrome de Burnout y factores asociados en estudiantes de medicina: Estudio multicéntrico en siete facultades de medicina peruanas. Rev. Chil Neuro-Psiquiatr. 2016; 54(3): 207-21.

23. Mayta-Tristán $P$, Cuentas $M$, NúñezVergara M. Responsabilidad de las instituciones ante la proliferación de escuelas de medicina en el Perú. Acta Med Peru. 2016; 33(3): 178-82.

24. Mayta-Tristán P, Poterico J, Galán-Rodas E, Raa-Ortiz D, El requisito obligatorio del servicio social en salud del Perú: discriminatorio e inconstitucional. Rev Peru Med Exp Sal Pub. 2014; 31(4): 781-7.

25. Albitres-Flores L, Loayza-Salvatierra N. Realidad de la Investigación en la Escuela de Medicina de la Universidad Nacional de Trujillo. Rev Med Trujillo. 2017; 12(1):3745.

26. Ramos J, González-Alcaide G, Gutiérrez F. Análisis bibliométrico de la producción científica española en Enfermedades Infecciosas y en Microbiología. Enferm Infecc Microbiol Clin 2016; 34(3):166-76. 